\title{
Latest 3-flavor neutrino oscillations results from the NOvA experiment
}

\section{Steven Calvez ${ }^{a, *}$}

${ }^{a}$ Colorado State University,

Department of Physics, 1875 Campus Delivery, Fort Collins, CO, USA

E-mail: steven.calvez@colostate.edu

NOvA is a long-baseline neutrino oscillation experiment. Its large tracking calorimeters can detect and identify muon and electron neutrino interactions with high efficiency. Neutrinos produced by the NuMI beam are detected by a Near Detector, located at Fermilab, and a much larger Far Detector, located $810 \mathrm{~km}$ away in Ash River, Minnesota. NOvA can measure the electron neutrino and antineutrino appearance rates, as well as the muon neutrino and antineutrino disappearance rates, in order to constrain neutrino oscillations parameters, including the neutrino mass hierarchy and the CP-violating phase $\delta_{C P}$. This talk will present NOvA's latest results [1] combining both neutrino data $\left(13.6 \times 10^{20} \mathrm{POT}\right)$ and antineutrino data $\left(12.5 \times 10^{20} \mathrm{POT}\right)$.

\footnotetext{
*** The European Physical Society Conference on High Energy Physics (EPS-HEP2021), *** *** 26-30 July $2021 * * *$

*** Online conference, jointly organized by Universität Hamburg and the research center DESY ***
}

\footnotetext{
${ }^{*}$ Speaker
} 
Long-baseline neutrino experiments like NOvA are designed to study the oscillations of neutrinos over long distances. Measuring accurately this phenomenon could help us determine the precise value of the mixing angle $\theta_{23}$, the sign of the mass splitting $\Delta m_{32}^{2}$ (i.e. the neutrino Mass Hierarchy), and potential CP-violation in the lepton sector. The latter property, in particular, could be a key ingredient towards explaining the matter-antimatter asymmetry observed in the Universe today.

\section{Principle of the NOvA 3-flavor neutrino oscillations analysis}

The NOvA experiment can detect both the disappearance of muon (anti)neutrinos and the appearance of electron (anti)neutrinos from a beam of muon (anti)neutrinos in order to constrain $\theta_{23}, \Delta m_{32}^{2}$ and a CP-violating phase $\delta_{C P}$ : by measuring the energy spectra of the muon neutrinos in a Near Detector, i.e. close to their source, and comparing it to the energy spectra of the surviving muon neutrinos and the appearing electron neutrinos, measured in a Far Detector, it is possible to assess these oscillation parameters. To first order, the survival probability of muon neutrinos depend on $\sin ^{2} 2 \theta_{23}$ and $\left|\Delta m_{32}^{2}\right|$, as expressed in Equation 1.

$$
P\left(v_{\mu} \rightarrow v_{\mu}\right) \approx 1-\cos ^{4}\left(\theta_{13}\right) \sin ^{2}\left(2 \theta_{23}\right) \sin ^{2}\left(\frac{\Delta m_{32}^{2} L}{4 E}\right)
$$

At NOvA's baseline, $L$, and neutrino energies, $E$, the effect of these parameters on the muon neutrinos energy spectra after oscillations is illustrated in Figure 1. While the disappearance channel provides us with strong constraints on the values of these parameters, it cannot help us determine the octant of $\theta_{23}$ (i.e. whether it is greater or less than $45^{\circ}$ ) nor the sign of $\Delta m_{32}^{2}$. However, this information, along with the value of $\delta_{C P}$, can be probed in the appareance channel, as evidenced by Equation 2. In addition, it is possible to further improve the measurement's sensitivity by combining both neutrino and antineutrino oscillation data, as illustrated by Figure 1 .

$$
\begin{aligned}
P\left(\stackrel{(-)}{v}_{\mu} \rightarrow \stackrel{(-)}{v}_{e}\right) & \approx \sin ^{2} 2 \theta_{13} \sin ^{2} \theta_{23} \frac{\sin ^{2}(A-1) \Delta}{(A-1)^{2}} \\
& \stackrel{(+)}{-} 2 \alpha \sin \theta_{13} \sin \delta_{C P} \sin 2 \theta_{12} \sin 2 \theta_{23} \frac{\sin A \Delta}{A} \frac{\sin (A-1) \Delta}{A-1} \sin \Delta \\
& +2 \alpha \sin \theta_{13} \cos \delta_{C P} \sin 2 \theta_{12} \sin 2 \theta_{23} \frac{\sin A \Delta}{A} \frac{\sin (A-1) \Delta}{A-1} \cos \Delta \\
& \text { Where: } \alpha=\frac{\Delta m_{21}^{2}}{\Delta m_{32}^{2}} \quad \Delta=\Delta m_{32}^{2} \frac{L}{4 E} \quad A=\stackrel{(-)}{+} G_{f} N_{e} \frac{L}{\sqrt{2} \Delta}
\end{aligned}
$$

\section{The NOvA experiment}

The NOvA experiment uses the high-purity muon neutrino NuMI beam (peak hourly average of $850 \mathrm{~kW}$ ) at Fermilab to send muon neutrinos to two functionally identical detectors, located slightly off the beam axis ( $2 \mathrm{GeV}$ energy peak). The Near Detector is located underground in Fermilab, while the much larger 14 kton Far Detector sits on the surface, $810 \mathrm{~km}$ further away, in Minnesota. 

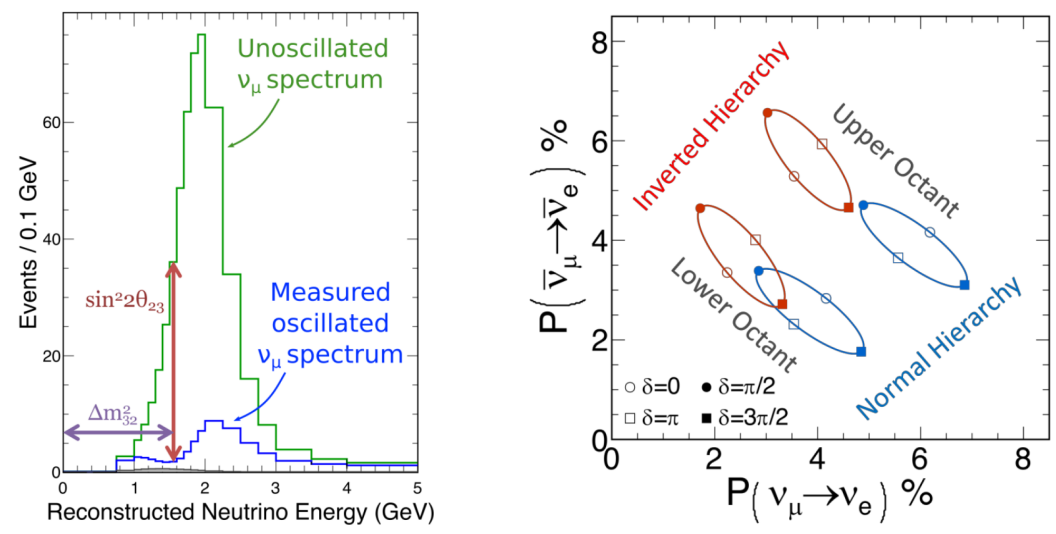

Figure 1: (left) Illustration of the effect of $\Delta m_{32}^{2}$ and $\sin ^{2} 2 \theta_{23}$ on the disappearing muon neutrino spectrum. (right) Effect of $\theta_{23}, \Delta m_{32}^{2}$ and $\delta_{C P}$ on the electron neutrino and antineutrino appearance probabilities.

They share the same experimental design in order to reduce the flux and cross-section systematics uncertainties. They are composed of $6.6 \mathrm{~cm} \times 3.9 \mathrm{~cm}$ plastic cells filled with liquid scintillator. The light produced by charged particles traveling through the detector is collected by wavelength shifting fibers and read out by avalanche photodiodes. This high granularity design allows NOvA to detect neutrino interactions, to identify individual particle tracks and to measure their energies. Different topologies of neutrino interactions are shown in Figure 2. Machine learning techniques are used to select candidate neutrino interactions and identify individual particles [2, 3]. The particles energies are reconstructed from the track length and the calorimetric energy deposits.

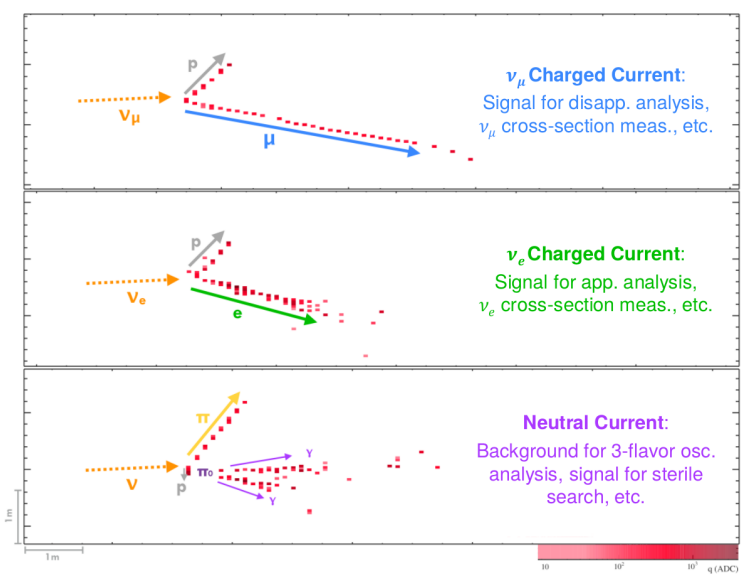

Figure 2: Event displays of different types of neutrino interactions as seen by the NOvA detectors.

\section{NOvA's latest 3-flavor neutrino oscillations results}

By combining both neutrino data $\left(13.6 \times 10^{20}\right.$ POT, $50 \%$ more than previous results [4] and antineutrino data $\left(12.5 \times 10^{20} \mathrm{POT}\right)$, and using improved analysis techniques and simulations, 
NOvA was able to set world-leading constraints on some of the least known neutrino oscillation parameters [1].

\subsection{Near Detector energy spectra}

The muon neutrino and antineutrino energy spectra measured at the Near Detector are presented in Figure 3. These measurements are pivotal to the analysis since extrapolating these spectra to the Far Detector permits us to greatly cancel out [1] the flux and cross-section uncertainties [5], which would otherwise be dominant. The statistical uncertainties on these spectra are very small, thanks to the NuMI high beam intensity. The main, and essentially only, background comes from wrong sign events, which are intrinsic to the beam and originate from the failure of the magnetic horns to focus the right charged hadrons, thus producing antineutrinos in a neutrino beam, and conversely. In practice, the extrapolation is performed in twelve separate subsamples, based on their energy resolution and transverse momentum, in order to improve the analysis sensitivity. In addition, since no electron (anti)neutrino appearance is expected in the Near Detector, measuring their energy spectrum provides us with a powerful estimate of the backgrounds for the appearance analysis. The predictions built by extrapolating both muon and electron (anti)neutrinos Near Detector spectra are then compared to the Far Detector data.
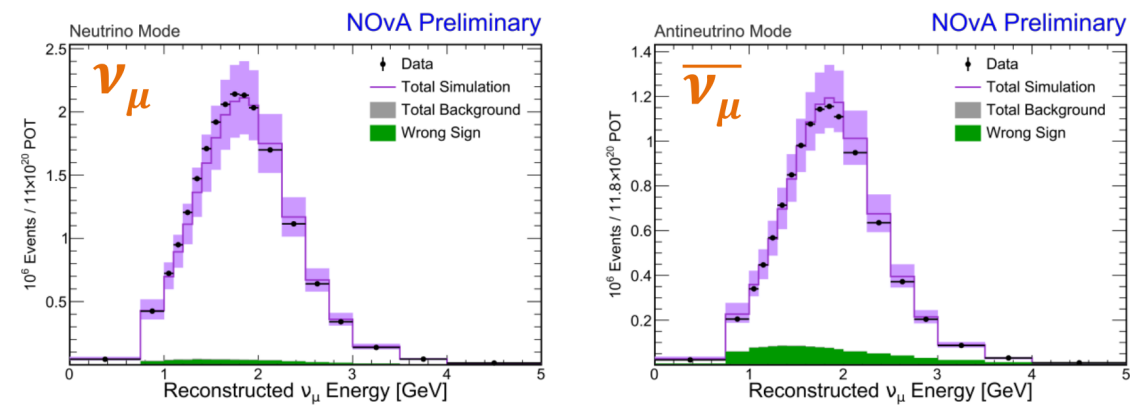

Figure 3: Muon neutrino (left) and antineutrino (right) energy spectra reconstructed in NOvA's Near Detector.

\subsection{Far Detector energy spectra}

The surviving muon (anti)neutrino energy spectra measured at the Far Detector are presented in Figure 4. The separate subsamples actually used in the analysis are also here shown merged for simplicity. In total, 211 muon neutrinos candidates have been selected while more than 1000 events would have been expected in the absence of oscillations. Similarly, 105 muon antineutrinos have been selected against around 500 without oscillations. The appearing electron (anti)neutrino energy spectra at the Far Detector are presented in Figure 4. In the analysis, the datasets are actually also further split in order to increase the measurement's sentivity, this time based on the confidence in the selection of an electron (anti)neutrino event. While no excess of events over the background, the colored spectra in Figure 4 (right), would have been expected in the absence of oscillation, NOvA observed 82 electron neutrino candidates and 33 electron antineutrino candidates. 

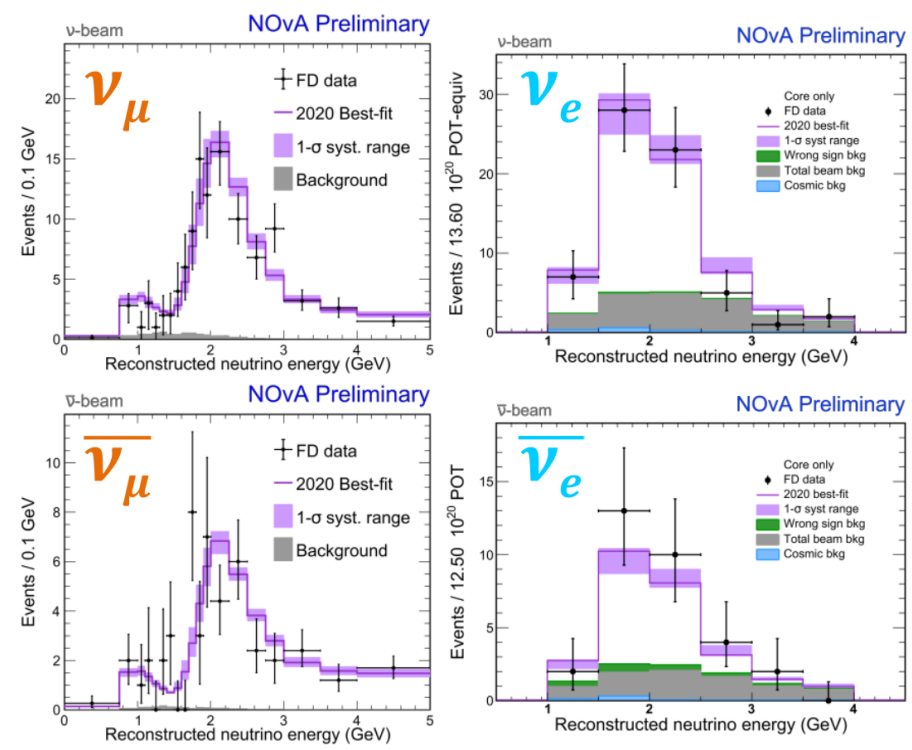

Figure 4: Surviving muon neutrino (top left) and antineutrino (bottom left) and appearing electron neutrino (top right) and antineutrino (bottom right) energy spectra reconstructed in NOvA's Far Detector.

\subsection{Constraints on the oscillation parameters}

The appearing and disappearing spectra in both neutrino and antineutrino mode are fitted together based on predictions built and extrapolated from the Near Detector. Confidence intervals are constructed based on the Feldman-Cousins Unified Method [6]. The significance for excluding values of the parameters $\sin ^{2} \theta_{23}, \Delta m_{32}^{2}$ or $\delta_{C P}$ are shown in Figure 5. The preferred regions of the parameter space for combinations of $\Delta m_{32}^{2}$ and $\sin ^{2} \theta_{23}$ or $\sin ^{2} \theta_{23}$ and $\delta_{C P}$ are presented in Figure 6. NOvA's best fit lies in the Upper Octant and the Normal Hierarchy. It has achieved a precise measurement of $\Delta m_{32}^{2}=2.41 \pm 0.07 \times 10^{-3} \mathrm{eV}^{2}( \pm 3 \%)$ and $\sin ^{2} \theta_{23}=0.57_{-0.03}^{+0.04}( \pm 7 \%)$. NOvA did not observe a significant asymmetry in $v_{e}-\overline{v_{e}}$ appearance and consequently disfavors regions around $\delta_{C P}=\pi / 2$ in the Inverted Hierarchy at more than the $3 \sigma$ confidence level and $\delta_{C P}=3 \pi / 2$ in the Normal Hierarchy at the $2 \sigma$ level. The data also points to a preference for the Normal Hierarchy at the $1.0 \sigma$ level, the upper octant at $1.2 \sigma$, and a non-maximal mixing at $1.0 \sigma$.
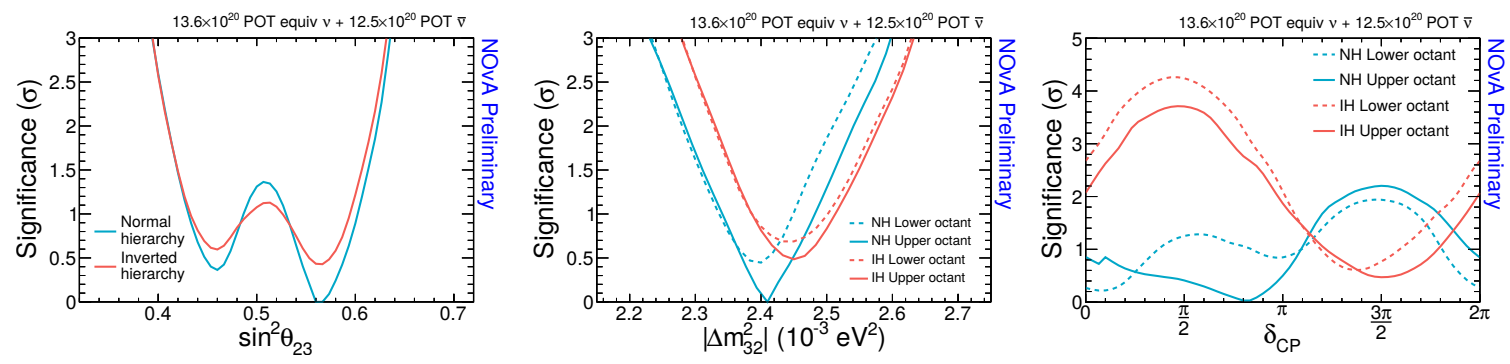

Figure 5: Exclusion significance for different values of $\sin ^{2} \theta_{23}$ (left), $\Delta m_{32}^{2}$ (middle) or $\delta_{C P}$ (right) in the Normal Hierarchy (blue) or the Inverted Hierarchy (red) and in the Lower Octant (dashed) or the Upper Octant (solid). 

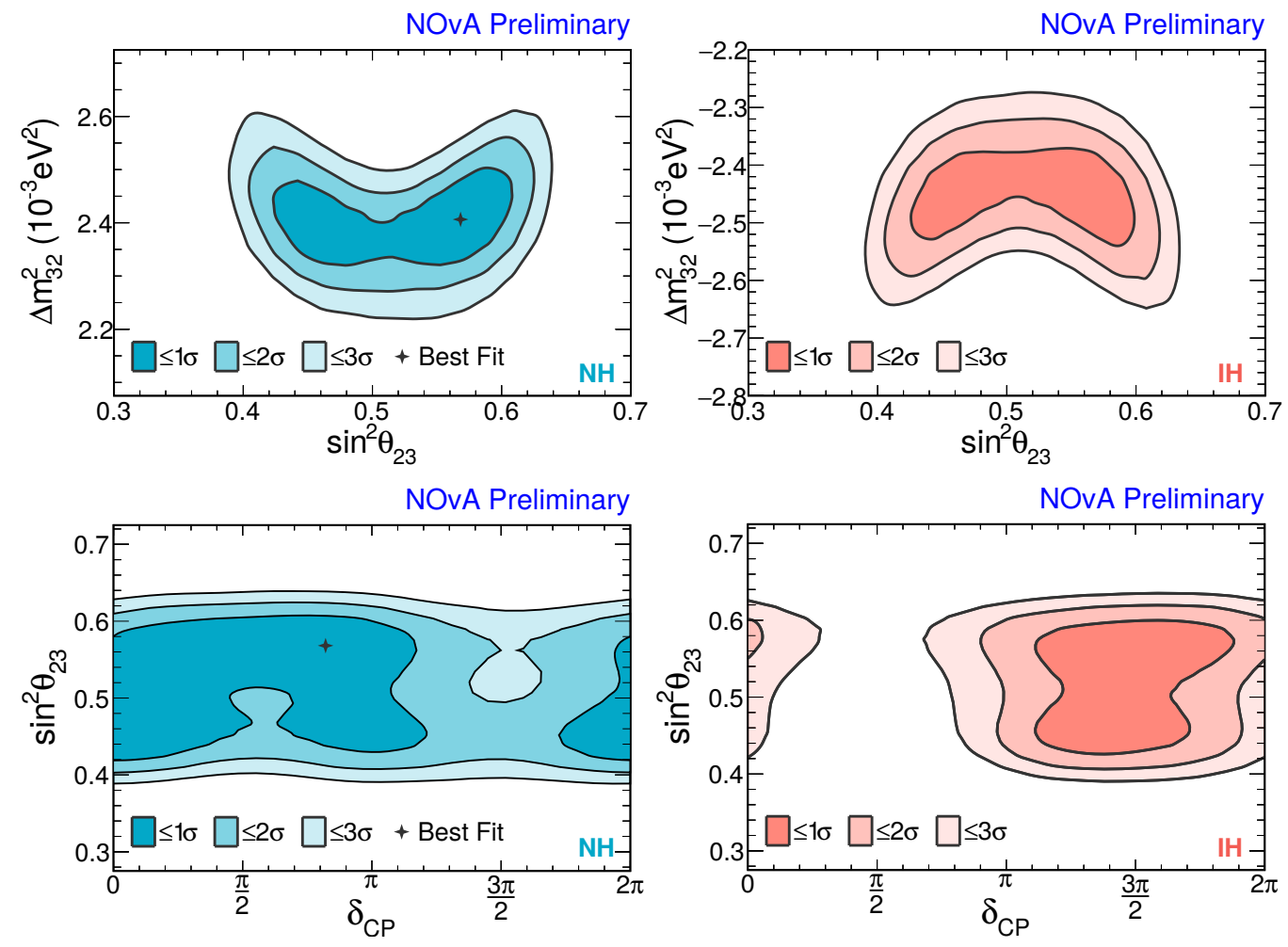

Figure 6: Confidence regions in $\Delta m_{32}^{2}$ vs. $\sin ^{2} \theta_{23}$ (top) and $\sin ^{2} \theta_{23}$ vs. $\delta_{C P}$ (bottom) in the Normal Hierarchy (left) or the Invervted Hierarchy (right).

\section{References}

[1] An Improved Measurement of Neutrino Oscillation Parameters by the NOvA Experiment, M. A. Acero et al., arXiv:2108.08219

[2] A convolutional neural network neutrino event classifier, A. Aurisano et al., JINST 11, P09001 (2016)

[3] Context-Enriched Identification of Particles with a Convolutional Network for Neutrino Events, F. Psihas et al., Phys. Rev. D 100, 073005 (2019)

[4] First measurement of neutrino oscillation parameters using neutrinos and antineutrinos by NOvA, M.A. Acero et al., Phys. Rev. Lett. 123, 151803 (2019)

[5] Adjusting neutrino interaction models and evaluating uncertainties using NOvA near detector data, M.A. Acero et al., Eur. Phys. J. C 8012, 1119 (2020)

[6] A Unified Approach to the Classical Statistical Analysis of Small Signals, G. J. Feldman and R. D. Cousins, Phys. Rev. D57, 3873 (1998) 